\title{
EL DISCURSO PSICOSOCIAL EN EL FUERO DE FAMILIA ESPAÑOL
}

\section{THE PSYCHOSOCIAL SPEECH IN THE SPANISH FAMILY COURTS}

\author{
FRANCISCO Gómez Gómez ${ }^{*}$ Raúl SOto EstebaN ${ }^{* *}$
}

\begin{abstract}
*Doctor y Licenciado en Ciencias Políticas y Sociología y Diplomado en Trabajo Social. Profesor Titular del Departamento de Trabajo Social y Servicios Sociales en la Facultad de Trabajo Social de la Universidad Complutense de Madrid.

E-Mail: fgomez@trs.ucm.es

Departamento de Trabajo Social y Servicios Sociales, Facultad de Trabajo Social Desp. 3110. Campus de Somosaguas 28223, Pozuelo de Alarcón (Madrid).

**Doctor en Trabajo Social y Licenciado en Historia del Arte. Trabajador Social de los Juzgados de Primera Instancia e Instrucción de Alcobendas en Madrid. E-Mail: raul.soto@algoasi.com
\end{abstract}

\section{REsUmen}

Los equipos psicosociales de la administración de justicia desde 1982 asesoran a los jueces en materia de conflictos familiares. El estudio que se preesenta informa cómo se ha elaborado el discurso de este colectivo, y parte de la noción de discurso de Michel Foucault, desde su visión problemática. Luego recoge, a través de entrevistas y grupos de discusión, las opiniones de los trabajadores sociales y psicólogos de Madrid y Barcelona sobre la gestión del discurso. El estudio plantea un supuesto inicial sobre un menor protagonismo de los trabajadores sociales en la creación del relato que corresponde a una práctica común y busca una explicación y una descripción de lo sucedido.

El trabajo que se presenta forma parte de una investigación académica básica y exploratoria de tipo cualitativo, basada en el principio fundamental de obtener los discursos principales para la resolución de los objetivos planteados como nuevas alternativas técnicas del trabajo psicosocial en los juzgados de familia y las tareas que de sarrollarán los equipos psicosociales con las familias.

Como conclusiones cabe resaltar que los trabajadores sociales forenses ven el futuro con optimismo, valoran su propia intervención en la ayuda a los jueces y creen que habría que impulsar una actuación profesional más propia. Los psicólogos y trabajadores sociales forenses entienden que hay un problema de falta de discurso psicosocial, por lo que es necesaria una mayor implicación en producir investigación en el área del discurso psicosocial. Aunque al evaluar la aportación de los trabajadores sociales la realidad resulta más pobre.

Palabras clave: Discurso psicosocial; Equipos psicosociales; Trabajo social judicial; Psicólogos; Juzgados de familia.

\section{ABSTRACT}

From 1982, psychosocial justice teams advise judges in family conflicts. This study explores how the discourse has been developed for this group. The article begins with the notion of discourse by Michel Foucault, from his problematic vision. Then, the views of social workers and psychologists on the management of speech, which have been collected through interviews and focus groups. The study raises an initial assumption of a lesser role of social workers in the creation of the story that 
corresponds to common practice and seeks an explanation and description of what happened.

The object of this research was to expound and to reveal the place of the professional expert social worker in the psychosocial teams of the admin istration of justice in (Gomez \& Soto 2015a). The social workers have an important role in the psychosocial teams in the administration of justice in the judges are at the receiving end of the social reports, the families are the object of this report and other professionals in the judicial system such as the psychologists, play their role in this team. Each professional group mentioned above requires a different type of task and a different assessment method: Social workers are the qualified profes sionals; the families are the object of the investigation, as they are the focus of the investigation. The opinion of the social worker is important and so is the perception of the situation of their professional group, in which they have the main role. The psychologists are the other professional group who serve the families and the courts during the investigation. Their perception of the discourse is valued as privileged witnesses. It is also interesting to note the significance of their contribution within this psychosocial collaboration.

The techniques to be implemented can be defined as group discussion: the format is open, the components must be homogeneous and heterogeneous at the same time. The researcher suggests the topic of discussion and does not intervene any more except to guide the meeting or to control aspects of the meeting. The recorded information is then analyzed and the group then proposes a discourse referred to as the popular social perception. The in-depth interview is a technique of the qualitative dimension and does not require excesive intervention from the researcher; the aim of the indepth interview is to draw a life history.

The research compared the professional real ities of forensic social workers in and from the view of psychologists and the social workers them selves. Psychologists were respected professionals of the social work, but they had important differences of opinion about the role they should play in the administration of justice and their relationships with the judges. The forensic social workers look at the future with optimism and value their inter vention in support of the judges; they feel that they should promote a more professional line of action within the lexica and techniques of social work playing a more important role in their activities. The psychologists and forensic social workers understand that there is a lack of psychosocial discourse and a greater involvement is required in the production of research and documented work within the psychosocial discourse. In particular, when the contributions of social workers are evaluated, this reality is lacking.

Key words: Psychosocial speech; Psychosocial teams; Court social work; Forensic psychologists; Family courts.

\section{INTRODUCCIÓN}

El término discurso, según la Real Academia Española (RAE, 2015), tiene varias acepciones, la primera es: "facultad racional con que se infieren unas cosas de otras, sacándolas por consecuencia de sus principios o conociéndolas por indicios y señales". La octava es: "Escrito o tratado de no mucha extensión, en que se discurre sobre una materia para enseñar o persuadir." Y la duodécima sería: "Carrera, curso, camino, que se hace por varias partes".

El significado de la palabra lleva consigo una intención, una manera de explicar la realidad. La dinámica psicosocial judicial ha tenido una historia de más de 30 años en la que un colectivo de profesionales públicos ha desarrollado su labor en la administración de justicia. Desde este escrito se valora la distinta participación del colectivo profesional de los trabajadores sociales y de los psicólogos forenses en la creación del discurso que ha acompañado a la práctica.

El pensador francés Michel Foucault planteaba que

"las prácticas discursivas se caracterizan por la delimitación de un campo de objetos, la definición de una perspectiva legítima para el agente del saber, y la fijación de normas para la elaboración de conceptos y teorías. Así, cada práctica discursiva implica un juego de 
prescripciones que designan sus exclusiones y elecciones" (1977, citado en Chambón, Irving \& Epstein, 2001, p. 370).

Pero además de describir este tipo de prácticas, Foucault hablaba de su dinamismo y su complejidad:

"hay que admitir un juego complejo e inestable donde el discurso puede, a la vez, ser instrumento y efecto de poder, pero también obstáculo, tope, punto de resistencia y de partida para una estrategia opuesta. El discurso transporta y produce poder: lo refuerza pero también lo mina, lo expone, lo torna frágil y permite detenerlo" (1992, en Chambón et al., 2001, p. 371).

Este estudio exploró el origen y la creación de los discursos, las palabras que se han usado y se usan para enseñar la materia Psicosocial. Es una etapa previa y exploratoria, en la que se exponen presupuestos nacidos del trabajo práctico de una disciplina ligada a la ciencia jurídica. Para llegar a la historia y a la forma en que se ha creado el discurso psicosocial dentro del ámbito judicial, este artículo presenta textos de profesionales protagonistas de esta creación. Las conclusiones contestarán al supuesto previo apuntado y darán materia de debate y estudio sobre la forma en que psicólogos y trabajadores sociales han participado de la explicación de su trabajo. No es una cuestión solo de delimitar el campo, también de conocer el contexto y, desde la asunción de la complejidad, valorar el juego de poder que todo discurso lleva consigo.

Los profesionales de los equipos psicosociales de los juzgados de familia nacen en España en 1982, con la promulgación de la ley del divorcio (Ley 30/1981, del 7 de julio, por la que se modifica la regulación del matrimonio en el Código Civil y se determina el procedimiento a seguir en las causas de nulidad, separación y divorcio) y la creación de estos juzgados de familia (De Andrés, 1997; Gómez, 1988). Psicólogos y trabajadores sociales han trabajado juntos desde ese momento y hasta la actualidad con dependencia pública y con integración en la administración de justicia de familia. No existe una regulación clara ni una integración de estas figuras profesionales en el procedimiento de familia, pero los jueces y fiscales de familia y los letrados han referido su importancia. El Fiscal López Urdiales (2008, citado en Gómez \& Soto, 2015a, p. 135) recoge la función pericial y añade funciones auxiliares de estos equipos:

"La actuación de los equipos psicosociales no sólo se ha de centrar en la elaboración del correspondiente informe técnico (...) sino que además podrán desarrollar otras complementarias como:

1.- Prestar ayuda puntual a la familia para afrontar la crisis familiar.

2.- Informar de la existencia de instrumentos alternativos para la solución de los conflictos relacionados con una crisis familiar, como lo es la mediación, que evitarían el procedimiento judicial contencioso y con los que se podrían obtener acuerdos sólidos y solventes.

3.- Otras labores de seguimiento y apoyo.

4.- Coordinación con los puntos de encuentro, etc."

Inicialmente el discurso es psicosocial; los profesionales exploran el nuevo campo y crean protocolos de actuación para integrarse al nuevo marco. Detrás de su trabajo hay una ideología profesional como así también técnicas; es una forma de trabajo que se realiza en equipo. Existen discursos que se explican entre los profesionales de la Psicología (Bolaños, 1993; Ibáñez \& De Luis, 1987) pero aunque escaso, también hay algún ejemplo de discurso del trabajo social (De Andrés, 1997).

Los psicólogos y trabajadores sociales conocen las ventajas de un trabajo en equipo, integrado y creador de una nueva disciplina mestiza:

"La colaboración interdisciplinaria es importante para mejorar la calidad en los servicios de atención a los pacientes, además, con este enfoque se genera mayor producción de ideas, metodología y resultados de investigación" (Woods \& Magyary, 2010, en Sierra Murguía, 2013). 
Ribes (2010, en Camacho, Arroyo y Serrano, 2011) lo define así:

"La vinculación entre dos o más disciplinas con fines tecnológicos o profesionales en el plano de la colaboración e intercambio de información para la solución de problemáticas de relevancia social."

Hay un momento en la historia en el que la situación cambia. Determinados psicólogos (Catalán, 1999; Fariña, Arce \& Sotelo, 2010) inician paralelamente un nuevo discurso individual, que además está acompañado con el nacimiento de alternativas formativas, que no se producen en el mundo del trabajo social; la universidad y los colegios profesionales empiezan a recoger y ofrecer cursos, seminarios o masters de Psicología Forense. Los psicólogos forenses españoles comienzan a hablar en otro lenguaje, más tecnificado e individual, individual no sólo en un sutil abandono de los compañeros de viaje y su aporte socio-familiar, sino también en la evaluación de los problemas familiares, objeto de su trabajo.

En Latinoamérica la situación se vive de otra forma y Quintero (2010) critica positivamente una situación de los equipos psicosociales en España que ella estima envidiable y estudia la situación en Colombia, llegando a conclusiones interesantes:

"Las pruebas psicosociales son un gran avance en el campo probatorio, ya que proporciona al operador jurídico un conocimiento indirecto sobre el hecho de estudio, en aras de una mejor comprensión" (p.76).

Otros trabajadores sociales (Alday, Ramljak \& Nicolini, 2001; Ponce de León \& Krmpotic, 2012; Robles, 2004) estudian la intervención del trabajador social en la administración de justicia argentina.

\section{OBJetIVOS}

Los objetivos que guiaron este estudio fueron los siguientes:

Plantear nuevas alternativas discursivas desde una mirada profesional del trabajo psi- cosocial. Describir las consecuencias de los diferentes discursos teóricos para este trabajo psicosocial en los juzgados de familia y explorar nuevas tareas a desarrollar con las familias por los equipos psicosociales.

\section{Metodología}

Se realizó una investigación básica y exploratoria que parte de un escaso desarrollo del discurso psicosocial dentro de los equipos técnicos de los juzgados de familia españoles y se preguntan a los protagonistas las razones de esta situación. Se buscan discursos para extraer significados que permitan generalizar y situar la teoría, a la vez que se avanzan nuevas alternativas en lo teórico y sus consecuencias en la práctica.

Por ello, la investigación es cualitativa y analiza los discursos desde el método comparativo constante de Glaser (Valles, 2007), el cual recoge los enfoques previos: uno destinado a la inspección de datos y otro, a la codificación. Propondría un tercer sistema combinado de ambos, en el que a través de la saturación de información se llegaría a la creación de teoría.

En primer lugar, el soporte informático (ATLAS-ti) hace anotaciones que se vinculan con el origen del estudio, luego en la medida en que se recogen estos datos se analizan, y finalmente a través de la codificación se formulan preguntas y se hacen comparaciones. Es lo que llaman grounded theory.

La metodología usa el sistema de Miller y Crabtree (1994, en Valles, 2007), que describe una escala de cuestiones a ser consideradas por el analista, asociadas a una mayor o menor descripción o a una posibilidad de concebir la teoría:

1.- El examen del problema y los objetivos del estudio. Si se persigue la exploración o la comprensión de experiencias ajenas, el analista deberá hacer uso de la estrategia que le permita estar más próximo al texto. Si el propósito es testear teoría, es preferible una estrategia más distanciada y estructurada. 
2.- La cantidad de conocimiento previo sobre la materia. A mayor cantidad de literatura científica o solidez teórica, mayor estructuración y distanciamiento.

3.- La coherencia con la técnica de obtención de datos.

4.- La coherencia con la perspectiva paradigmática en la que se inserte el estudio.

En el caso de esta investigación, es adecua do acercarse al texto para describir, considerando la escasez de textos previos y dentro de una lógica de inmersión en la cuestión, que permite este tipo de análisis. En el proceso van a obtenerse discursos principales para la resolución del objetivo y la información va a saturar el estudio por el número de participantes y la condición de expertos de los entrevistados y llamados a debatir en grupos de discusión.

\section{PARTICIPANTES}

La investigación priorizó las provincias de Madrid y Barcelona como muestra. La potencia demográfica de estas dos principales ciudades del estado, permite la agrupación de profesionales, la gestión grupal de necesidades y la producción documental, es por este motivo que ahí se desarrolló el trabajo de campo. Participaron ocho psicólogos y cinco trabajadores sociales de los equipos psicosociales del SATAF de Barcelona y seis trabajadores sociales y cuatro psicólogos de los equipos psicosociales de la Comunidad de Madrid que realizan su trabajo en dichas ciudades.

Se trabajó con 16 psicólogos y 8 trabajadores sociales de Barcelona y 43 psicólogos y 34 trabajadores sociales de la Comunidad de Madrid, lo que da un porcentaje de un $32 \%$ de la muestra total de técnicos de estas provincias en ese momento de la investigación (32 técnicos explorados de un total de 101).

Los profesionales de Barcelona desarrollaban su labor en un mismo lugar (en el momento de la exploración) y un mismo domicilio, mientras los madrileños se distribuían en los distintos juzgados de la capital o del extrarradio (Gómez \& Soto, 2015b).

\section{INSTRUMENTOS}

El análisis de los discursos es un proceso de investigación cualitativa en el que se buscan significados y se ordenan de acuerdo a los objetivos.

La metodología es doble: la entrevista abierta a psicólogos y trabajadores sociales de la administración de justicia y la realización de grupos de discusión triangulares de psicólogos y trabajadores sociales en la ciudad de Madrid (dos grupos de trabajadores sociales y uno de psicólogos, de tres personas cada uno).

\section{Procedimiento}

Las entrevistas y los grupos de discusión triangulares se llevaron a cabo entre noviembre de 2010 y julio de 2012. Los discursos de los profesionales explorados se agruparon en una unidad hermenéutica, luego se inició el proceso de codificación abierta de acuerdo a Valles (2007), buscando categorías que ayudaran a comparar. Se codificó según las profesiones de las personas entrevistadas o convocadas a grupos y también según las variables que asociadas a los objetivos del estudio permitieron una interpretación de los discursos. Esta interpretación siguió el criterio de parsimonia o economía científica, por el que se hizo máxima una explicación científica con un mínimo de conceptos o formulaciones.

Para una mejor identificación de los discursos, en cada cita se identifica el lugar geográfico, el profesional y también se distingue su aporte, sea desde una entrevista o su participación en un grupo de discusión.

\section{VARIABLES}

Las variables estudiadas son las siguientes: 1.- Historia profesional común con reconocimiento mutuo.

2.- Distinta implicación de ambas profesiones en la elaboración del discurso psicosocial. 
3.- Existencia de discursos aislados y de pocos creadores de discurso.

4.- Planteamiento de un doble camino: psicosocial o psicológico individual.

5.- La imagen pública de las profesiones.

6.- Las instituciones del trabajo social y la Psicología.

7.- La importancia de la formación y la investigación.

8.- El rango académico.

9.- El lugar y rol auxiliar del trabajo social.

10.- El carácter generalista del trabajo social.

11.- La falta de autoestima profesional.

12.- La condición femenina del trabajo social.

13.- La mirada al futuro.

\section{ANÁLISIS DE DATOS}

La investigación trazó el mapa de situación del discurso psicosocial desde la interpretación de los discursos de los profesionales en relación a las variables destacadas:

\section{1.- LA CONSTATACIÓN DE UNA HISTORIA PROFESIO- NAL COMÚN Y DE UNA LABOR CONJUNTA EN LA QUE SE DA UN RECONOCIMIENTO MUTUO}

Es una variable destacada en los profesionales de Barcelona. Ellos entienden que el trabajo de evaluación de las familias inmersas en crisis matrimoniales y que pedían al juez una respuesta a su situación, era una labor a desempeñar de forma interdisciplinaria. Los psicólogos entrevistados refieren un respeto y una satisfacción por la tarea realizada. Los profesionales más antiguos emiten un discurso orgulloso sobre la creación de una nueva realidad profesional.

"El planteamiento vino dado por la historia previa de formación, desde el primer momento vimos que la historia tenía que ser psicosocial, que los equipos estaban formados por psicólogos y asistentes sociales, por algo, porque era el tipo de intervención que se tenía que hacer en el campo de la familia, de diagnóstico. Y, bueno, se planteó intervenir, hacíamos las entrevistas conjuntas psicólogo y trabajador social, cada uno desde su lectura, desde su perspectiva, desde la intervención de su campo y posteriormente los informes los hacíamos también conjuntamente, cada uno desde su perspectiva" (Octavo psicólogo de Barcelona entrevistado, comunicación personal, 31/1/2011; Cita de PS8B).

Por otro lado, en Barcelona se trabaja en la creación de protocolos e investigaciones y es una labor común en la que se destaca la mezcla de disciplinas.

"Aquí hemos hecho trabajos juntos, en la mayoría de las investigaciones hay psicólogos y trabajadores sociales" (Tercer psicólogo de Barcelona entrevistado, comunicación personal, 27/1/2011; Cita de PS3B).

Y hay quien divide el trabajo y explica la forma en que cada disciplina ha sido importante para lo conseguido, para el lugar en la historia de los conflictos familiares que tienen los equipos psicosociales.

"No lo sé, lo que sí sé es que los protocolos que hemos hecho en Cataluña, son protocolos que son como son porque hemos tenido a los trabajadores sociales. Los psicólogos contamos las cosas a nuestra manera, todo es la personalidad, el desarrollo... El punto de vista social es muy importante y a veces se nos olvida, y a veces ellos mismos se quejan de que no se les tiene en cuenta y aquí nuestros trabajadores sociales son bastante refunfuñones y reivindicativos, aunque a veces no se distingue" (Sexto psicólogo de Barcelona entrevistado, comunicación personal, Cita de PS6B).

La sensación del trabajo en común también tenía una orientación, una ideología en la que se eligió que los hijos tenían padre y madre a pesar de que se separasen y que la relación de cada uno de los padres y los hijos debía continuar. Existía en el principio del ejercicio de la ley (años 80) una visión diferente en algunos operadores jurídicos sobre la visión del divorcio como una cuestión de progenitor culpable y progenitor inocente y la 
atribución del cuidado de los niños según esa calificación previa.

"La orientación siempre iba encaminada a que los niños tenían padre y madre y que tenían que conservar a ambos, esto costó mucho que fuera aceptado, porque la idea que había era de buenos y malos, uno era bueno y otro era malo, entonces la idea de que era una conflictiva de dos adultos en la que se implicaba a los menores y había que dejarlos aparte" (Cita de PS8B).

También es importante constatar que esta forma de trabajar que defienden los psicólogos de Barcelona, no es la única. Hay profesionales que han defendido el trabajo disgregado, totalmente separado. Esta situación se refleja en las opiniones de algún profesional, como en las de esta trabajadora social que critica la postura.

"Lo único que ya no me ha gustado es que últimamente, no últimamente, no, hace un período de tiempo no muy lejano, pero no últimamente, ha habido una corriente que creo que aquí no ha pasado, que aquí en Madrid capital no ha pasado; esa corriente de los psicólogos por un lado, los trabajadores sociales por el otro, tú no te metas en mi trabajo. Eso no me parece bien, me parece feo, de muy mal gusto, de profesionales que no tienen ninguna entidad, que tienen miedo, que tienen inseguridad, porque todo lo que sea trabajar con una persona al lado que te enriquece" (Primer trabajador social de Madrid entrevistado, comunicación personal, 16/12/ 2010; Cita de TS1M).

2.- LA AFIRMACIÓN DEL SUPUESTO PREVIO: UNA DISTINTA IMPLICACIÓN DE CADA UNA DE LAS PROFESIONES EN LA ELABORACIÓN DEL DISCURSO PSICOSOCIAL

En las entrevistas es común que los psicólogos reconozcan no haber leído y ni siquiera conocen algún texto de trabajadores sociales judiciales, mientras que los trabajadores sociales judiciales se quejan de la au- sencia de literatura propia y describan la creada por los psicólogos como su fuente de inspiración a nivel forense.

"En la psicología hay mucho campo, pero desconozco en trabajo social. Entonces más que una hipótesis, estar de acuerdo o no, es un hecho claro, no hay ese crecimiento teórico en el trabajo social, pero no te sabría explicar porqué, porque efectivamente están ahí trabajando también, desde el principio" (Tercer psicólogo de Madrid entrevistado, comunicación personal, 29/11/2011, Cita de PS 3M).

"Sí que creo que todo ese cuerpo teórico sobre la intervención y la metodología, ha sido creado por psicólogos. No tengo una opinión general porque yo estoy circunscrito a la intervención con mi compañera, entonces yo creo que después de 14 años y medio trabajando de forma conjunta, creo que una metodología conjunta se podría hacer por parte de los dos colectivos en situación de igualdad, lo que sí creo es que no es nuestro caso, pero que en temas de una metodología conjunta, la fígura del trabajador social está algo marginada en algunos casos" (Segundo psicólogo de Madrid entrevistado, comunicación personal, 29/11/2011, Cita de PS2M).

Es evidente la perplejidad con la que algún profesional responde a esta pregunta, es algo que sucede pero en lo que no parecía haber caído antes.

"La verdad es que no te sabría decir porque no lo he pensado detenidamente. Ahora que lo dices es cierto, la mayoría de lo que hemos leído, cosas de evaluación y de propuestas en este ámbito, sí que es verdad que proceden más de psicólogos que de trabajadores sociales" (Cuarto psicólogo de Madrid entrevistado, comunicación personal, 29/11/ 2011, Cita de PS4M).

Los psicólogos de Madrid son los que confirman este supuesto con mayor profundidad, mientras los de Barcelona indagan en las causas sin reforzarlo. Los trabajadores socia- 
les de ambas localidades lo valoran como respuesta inmediata, sin cuestionarlo.

La situación es también desigual si se buscan cursos, master y estudios sobre la evaluación psicosocial de familias en crisis matrimoniales. Las facultades de Psicología, los colegios profesionales de Psicología y distintas entidades privadas ofrecen cursos y master de Psicología Forense (el listado sería largo y poco útil). Las instituciones de trabajo social apenas recogen algún curso de especialista en los colegios profesionales, orientados sobre todo a cumplimentar la obligación legal con respecto a los listados profesionales para elaborar periciales privadas.

\section{3.- LA DESCRIPCIÓN DE DISCURSOS AISLADOS, NO UN DISCURSO ÚNICO. Y LA ESCASEZ DE CREADORES DE DISCURSO}

Hay una crítica a la falta de unificación del discurso, a la diversidad exagerada de las opiniones profesionales, pero en esta ocasión se vuelve a observar cómo son los psicólogos, los que opinan sobre la pobreza de la literatura generada, y cómo dentro de esa penuria se destacan profesionales que son psicólogos de profesión. Los trabajadores sociales asienten nuevamente a esta opinión y no se aprecia una adscripción a ningún tipo de discurso en los trabajadores sociales.

"Discurso nuestro no, hay profesionales individuales (...) que han publicado cosas, y que pueden documentarse, no conozco ninguno de trabajadores sociales, no sé si existe. (...) Yo sé que los psicólogos que empiezan se documentan, el libro de Marta Ramírez como es muy práctico, es de una orientación propia. Luego sí que hay gente que ha creado discurso, esta gente de Santiago (...), es un discurso de actuación metodológica, muy de custodia compartida, muy de cómo hay que trabajar, hay que pasar pruebas, son muy directivos. Marcan una manera de trabajar y eso a lo mejor sí que ha creado discurso, no sé" (Cita de PS1M).

De nuevo se dan referencias al pasado, a una situación ideal para quien la describe que algunos profesionales vieron en Barcelona en los primeros años de trabajo psicosocial.

"Como colectivo, (...) en Barcelona es lo que se pretendía hacer, me da la impresión que ahora hay mucho de cara a la galería: página Web del SATAF. Veo que se han quedado en esquemas, hacemos esto y ya está. Aquello de aquellos años, era más ambicioso, inducido a lo mejor por Ignacio Bolaños que estaba allí, estaba interesado en teorizar, (...) la supervisión. Había una idea de qué cosas hacemos diferentes y porqué, y parte del discurso que se intentaba hacer, era a construir un poco lo de la mediación" (Cita de PS1M).

Pero la autocrítica es general, se insiste en la diferencia de discurso, pero desde la falta, desde la escasez. También se apunta el mayor poder y la mayor presencia de la Psicología y sus instituciones, dentro de una falta de propuestas.

"Fíjate, yo de todas formas te diría que hemos sido un colectivo, tanto unos profesionales como los otros, poco empeñados en los aspectos metodológicos, un poco, entonces a lo mejor no hay tanta diferencia en lo que hemos ido difundiendo unos y otros. Nuestra profesión y nuestro colegio tienen más visibilidad, y en ese sentido, tiene más accesibilidad a medios y demás, tampoco para tirar cohetes, pero algo más que pueda tener la vuestra, entiendo. No te sabría decir, yo creo que hemos sido un colectivo que hemos hecho pocas propuestas para poner a prueba, parco o vago" (Cita de PS4M).

El problema es que hay una gran fuente de datos primarios que se está desperdiciando por esa ausencia de iniciativa. Las entrevistas y exploraciones en situaciones en crisis generan dinámicas familiares susceptibles de estudio y seguimiento más allá de la evaluación pericial.

"¿Yo muchas veces digo con todos los datos tenemos hacer algo? No se entiende, pero hay gente que mira para otro lado. Nos estamos desperdiciando, podemos por edades, 
por situaciones, por conflictos, una cantidad de variables que se nos pierden que podíamos poner en común, tanto el trabajador social como el psicólogo, para hacer hipótesis y mejorar el trabajo con el usuario. Sí que es verdad que nos llevamos bien, somos políticamente correctos y nadie se atreve a pasar este tema" (Tercer psicólogo de Madrid entrevistado, comunicación personal, 29/11/ 2011, Cita de PS3M).

\section{4.- El PLANTEO DE UN DOBLE CAMINO: El PSICO- SOCIAL O EL PSICOLÓGICO INDIVIDUAL}

Esta variable enlaza con la preocupación por el enfoque del trabajo, observada en la cita referida al inicio profesional de lo psicosocial en Barcelona, pero late en otras muchas intervenciones. Existe una idea inicial de trabajo con orientación sistémica y con metodología psicosocial, sobre todo en Barcelona y al principio, que parece el exponente claro de esta visión del trabajo. Hay muchos profesionales influidos por este tipo de trabajo, con conexiones con la terapia familiar y con la teoría sistémica. Posteriormente, ha surgido un grupo de profesionales formados en distintos master o cursos de experto en las facultades de Psicología, los cuales defienden la Psicología Forense y la forma de trabajo basada en análisis individuales más que en estudios familiares. Aunque es excesivamente simplista, el análisis puede valer para entender el relato que plantea esta variable como fundamental.

"Está tan de moda hacer muchos cursos de Psicología Forense, de tantas horas, yo creo que esto con veinte horas lo explicas, yo creo que lo que hay que saber es Psicología Clínica, y eso se favorece menos. La vertiente que está de moda es la Psicología Forense, entonces se aborda la profesión desde una perspectiva, como te lo diría, defensiva, desde un punto de vista psicoanalítico. Porque todo lo que digas el señor o la señora porque lo di ce o no lo dice, es una visión que genera... Son la gente que (...) tiene una formación que son muy buenos a ese nivel pero que van, parten del hecho de que todos mienten, y a mí me cuesta entrar en esta dinámica, pero a lo mejor soy yo la que está equivocada. Vendría mejor saber cómo funciona la depresión o que problemas tiene la separación, más Psicología Clínica Sistémica, algo más de normal. Luego es verdad, que en familia lo que vemos son vivencias normales, pueden ser exacerbadas, que se hayan salido de madre, pero no dejan de ser procesos desgraciadamente normales dentro de lo que es la separación, el desamor" (Cita de PS1M).

\section{5. - LA IMAGEN PÚBLICA DE LAS PROFESIONES}

Los psicólogos de Barcelona evalúan la imagen de ambas profesiones y aprecian una diferencia clara. Es evidente que la situación de los trabajadores sociales es diferente, la cuestión es por que se ha dado esta diferente evolución profesional cuando el origen es similar.

"El psicólogo ha ido ampliando y se ha abierto el espectro a que el psicólogo pueda estar en casi todas las partes, y en este proceso el trabajador social también está haciendo un proceso parecido de ver en todos los lugares en que puede estar ya sea a nivel público o a nivel privado, (...). Entonces, si estamos en esto, el trabajador social en cuanto a abrir este espectro, incorporar el ámbito judicial quizás ha sido algo posterior" (Segundo psicólogo de Barcelona entrevistado, comunicación personal, 27/1/2011, Cita de PS2B).

Se compara desfavorablemente a los trabajadores sociales y se añade una observación sobre la visión que los jueces puedan tener sobre ambas profesiones y su utilidad. Esto conecta con la forma en la que las distintas profesiones han defendido un campo propio de trabajo.

"Sois un animal en peligro de extinción", eso se lo digo, "es que no lo veis, vais a desaparecer", (...) pero las valoraciones pienso que los jueces les valoran más, esto es como el valor en la mili, se les supone y seas malo, 
bueno o regular, pero psicólogo. Y eso se supone, como a un nivel de coordinación, que si eres psicólogo puedes hacerlo todo. Y en cambio si eres trabajador social solo puedes hacer un ámbito. El psicólogo abarca su línea psicológica pero también puede hacer valoración social, el trabajador social, solo valoración social. Si yo soy juez digo, pues le pido a uno que me lo haga todo, para eso están, esa es mi teoría" (Séptimo psicólogo entrevistado, comunicación personal, 28/1/2011, PS7B).

Los psicólogos en Madrid reconocen con menor crudeza la diferencia y hablan de un mayor prestigio social de su profesión, dentro de un ejercicio laboral similar.

"La idea que existe es esa, de cierta marginación en el abordaje de los casos, pero no solo aquí dentro, también fuera en la sociedad, se da más importancia al psicólogo que al trabajador social, pero incluso ejerciendo las mismas funciones, o haciendo las mismas investigaciones o usando las mismas fuentes de información" (Cita de PS2M).

La visión desde el trabajo social es amar ga, pero refuerza la importancia de la imagen pública para la gestión del discurso. El camino lo han empezado a andar los psicólogos y nadie les ha impedido a los trabajadores sociales hacer el suyo, no hay recriminaciones en el colectivo pero sí una conciencia de cierta desigualdad en la consideración pública de cada profesión.

"Tienen mucha práctica hecha. Yo leía mucho al empezar y si escriben los psicólogos bien, y si lo hacemos los trabajadores sociales es que copiamos a los psicólogos. Esta era la visión de entonces y no sé si no ha seguido, se podían escribir cosas, pero por lo que fuese el mayor reconocimiento social era de ellos. Yo creo que tiene una connotación social, estamos en una sociedad jerárquica" (Quinto trabajador social de Barcelona entrevistado, comunicación personal, 31/1/2011, Cita de TS5B).

La visión es constructiva, el trabajo se sigue realizando y sigue existiendo la necesidad de escribir y sistematizar la práctica, investigar para mejorar. Además en el trabajo diario es preciso tomar protagonismo, hacer el trabajo que corresponde sin ocultarse tras otros profesionales.

"Pero mi opinión personal es que tenemos que coger las riendas, dar a conocernos más, nuestra aportación a este trabajo y a lo mejor ayuda a romper de forma drástica con tabúes, vicios que se arrastren. Como que una pericial no la podamos hacer solo un trabajador social. No es una cosa individual el que tú tengas que llegar ahí, tiene que ser a nivel de colectivo. Necesitamos vernos más, no tenemos un contacto, el que tendríamos que tener, ni un espacio, ni un intercambio" (Sexto trabajador social de Madrid entrevistado, comunicación personal, 14/10/2011, Cita de TS6M).

\section{6.- El PAPEL dE LAS INSTITUCIONES: El COLEGIO PROFESIONAL Y LAS FACULTADES O ESCUELAS UNI- VERSITARIAS. LA DEBILIDAD DE LAS INSTITUCIONES DEL TRABAJO SOCIAL}

En muchas intervenciones se refiere la trascendencia de las instituciones en la redacción de los discursos de cada profesión. Los psicólogos de Barcelona critican la situación de los colegios profesionales, pero al margen de la crítica refieren una mayor actividad y ocupación del ámbito judicial en el de psicólogos.

"Yo tengo la sensación de que tiene que ver con los colegios respectivos, básicamente. Aquí en Barcelona aunque yo muestro mi disconformidad con el Colegio de Psicólogos sí que se mueven mucho más, que no el de trabajador social" (Primer psicólogo de Barcelona entrevistado, comunicación personal, 27/1/2011, Cita de PS1B).

"Los colegios profesionales, no podría decir cual funciona bien, como para coger a sus profesionales y relanzarles. No sé como irá el de trabajadores sociales pero me imagino que renqueando si hay" (Cita de PS1B). 
En el año 2008 en Madrid se ha constituido un grupo de trabajo dentro del Colegio Oficial de Trabajadores Sociales, que trabaja los temas de justicia; a este grupo y su nueva influencia alude alguno de los profesionales como impulsor de formaciones o estudios sobre el trabajo social judicial. Y se incide en la responsabilidad de la Escuela Universitaria de Trabajo Social (hoy Facultad) para formar en este ámbito.

"No, no ahora porque existe la formación más específica, que ha dado el grupo de justicia (...) la escuela tiene su responsabilidad, las prácticas, o por ejemplo el colegio, no sé ahora, pero antes ha pasado ampliamente toda la vida" (Cita de TS1M).

Los trabajadores sociales de Madrid y Barcelona coinciden e insisten en la necesidad de que la universidad y los colegios profesionales formen en trabajo social judicial y habiliten protocolos para la realización de prácticas en los juzgados. También comparan su situación con la gestión realizada por las facultades de Psicología o los colegios profesionales de esta disciplina que han promovido master o cursos de especialistas en Psicología Forense.

"Los otros son de intelectualizar y llevar a teoría. Tiene que ver los master que hay, luego las prácticas, que hay aquí en familia, el de la Complutense y el del colegio que hace master en forense. Luego, tienen prácticas y eso hace que también... Los trabajadores sociales no tenemos master, ni prácticas" (Segundo trabajador social de Barcelona entrevistado, comunicación personal, 27/1/2011, cita de TS 2B).

\section{7.- LA IMPORTANCIA DE LA FORMACIÓN Y DENTRO DE ELLA, EL ÉNFASIS EN LA INVESTIGACIÓN}

Los psicólogos destacan como propia esa preocupación por investigar. Hay una educación en la necesidad de ser científicos y crear saber:

"Yo en la universidad ya decían qué es lo primero, hay que avanzar en ello, en investi- gar. Luego se cayó en una dinámica que todo se investigaba, $\mathrm{N}=1$ y se investiga" (Cuarto psicólogo de Barcelona entrevistado, comunicación personal, 27/1/2011, cita de PS4B).

Los profesionales con experiencia hablan de un trabajo escaso y con poca continuidad.

"Y luego con las escuelas yo di formación en algún momento, pero... Yo creo que sí debería haber más presencia. Luego creo que sí ha hecho cosas el colegio, dando pasos, algo me ha ido llegando, sobre la imagen y presencia del trabajo social, yo te hablo de hace veinte años. (...) Consolidado cosas, y que hay profesionales más jóvenes que quieren reivindicar cosas para el trabajo social" (Cita de TS5B).

Los psicólogos dan importancia a la formación continua y ven en su profesión y sus instituciones canales para ejercer esa formación. Es una sensación de necesitar más herramientas que no aprecian en sus compañeros de trabajo.

"En los compañeros psicólogos sí que he visto la necesidad de formación, cuando han entrado compañeros nuevos sí que han buscado como formarse, que pruebas, puedo ir a verte, y la necesidad esa en Psicología Forense que enseguida te das cuenta, que te quedas cojo si no la tienes, y sin embargo lo que me ha pasado con los trabajadores sociales que he tenido nunca he tenido esa sensación. (...). No me han transmitido esa sensación de necesitar más formación, cosa que no pasa en el ámbito de la Psicología, a lo mejor los inseguros somos nosotros, no sé" (Primer psicólogo, grupo de discusión psicólogos, comunicación personal, 13/7/2012, GDPS).

En los debates de los grupos de discusión, en concreto en el de psicólogos se discute si puede existir una cierta inseguridad en esa búsqueda de formación continua. Es una situación diferente.

"Pero sí que veo que nosotros tenemos la sensación de tener que seguir haciendo cur- 
sos, formándonos continuamente, ahora con la acreditación de psicólogo forense que estuve trabajando en el COP, es una complicación. Al final te tienes que estar formando continuamente, todos los años demostrando al COP que tienes años de formación, no sé... Yo desde luego en los compañeros trabajadores sociales no he visto nunca" (GDPS).

Los trabajadores sociales tienen en claro que la razón es formativa y que parte del origen académico, que hay que indagar en la razón de ser para que los trabajadores sociales no sean investigadores de su propia práctica.

"Yo creo que también la gente debe tener formación de otro tipo porque ayuda. Y hasta ahora lo que he visto es que el trabajador social se quedaba con los 3 años y a buscar trabajo. Y los psicólogos en seguida quieren ampliar el campo, y entonces eso les hace ser más críticos con todo, hablar de todo etc....

No se nos forma, no se nos dirige al tema de publicar, escribir, o investigar. A otras disciplinas sí que se les dirige. Y la práctica, el día a día, te lleva, sabes que lo sabes y te dejas llevar. Tampoco lo ves una necesidad" (Primer trabajador social, primer grupo de discusión trabajadores sociales, comunicación personal, 29/3/2012, GDTS1).

\section{8. - El RANGO ACADÉMICO}

Los psicólogos y trabajadores sociales sitúan esta variable y con distintos matices la opinión es general, la diferencia académica es muy importante, sobre todo en un ámbito co mo el judicial en el que las jerarquías cobran gran importancia y en el cual la credibilidad del perito se basa en criterios de competencia.

"Para mí (las razones) son varias: Primero somos una carrera media y la de ellos superior, segundo el perfil del trabajador social es más asistencial que el del psicólogo" (Sexto trabajador social entrevistado, 14/10/2011, comunicación personal, Cita de TS6M).

"Yo creo que esto va más ligado a la diplomatura, a la trayectoria histórica acadé- micamente, lo asistencial se arrastra" (Cita de TS1M).

"Yo añado dos cosas, es personal. En el ámbito judicial en el que estamos que es muy jerarquizado, el hecho de que el trabajo interdisciplinar tuviera una jerarquía diferente: unos licenciados y otros diplomados ha sido malo. Yo estoy contenta de que vayamos a ser grado y dentro de 10 años esto se verá de otra manera. Estoy casi segura de que cuando se conozca por jueces y fiscales se va a ver de la misma manera" (Primer trabajador social, segundo grupo de discusión trabajadores sociales, comunicación personal, 49/5/2012, GDTS2).

Los psicólogos también lo ven claro y en su grupo de discusión argumentan que la diferencia académica es un criterio de discriminación.

"No sé si eso también influye a la hora de trabajar dentro de los equipos: la diferencia entre una diplomatura y una licenciatura, la diferencia de sueldo, no sé. Y eso unido a cómo se puede sentir la persona, creo que es algo que puede afectar, que dificulte a que haya una coordinación dentro del equipo, como una igualdad a la hora de trabajar (...). Y no tanto por el sentimiento de inferioridad que pueda tener un trabajador social, sino también por el sentimiento de superioridad que pueda tener el psicólogo" (GDPS).

"Yo pienso que también hay componentes sociales y culturales en este tema, hay disciplinas que por ser diplomaturas, (...) son consideradas a otro nivel desde otros ámbitos, no digo compañeros, sino facultades y otros, como inferiores entre comillas" (GDPS).

Finalmente se apunta que el cambio de categoría académica de los trabajadores sociales puede ayudar a una nueva visión:

"Yo creo que el tema del grado va a ayudar" (GDTS1).

"Es un camino que han hecho nuestros compañeros con el grado. Que según investigas sobre lo que se está trabajando" (GDTS2). 


\section{9.- El CARÁCTER GENERALISTA DE LA PROFESIÓN DEL TRABAJO SOCIAL}

Habitualmente hay una referencia común en la profesión de trabajador social hacia el carácter generalista, la escasa especialización y la característica de ser buenos gestores para el conocimiento general y ser capaces para el trabajo común, en equipo. Cuando el trabajo se hace específico se diluye esta característica y la labor profesional exige otras capacidades.

"Yo creo que al trabajo social judicial le ha pasado lo que le puede pasar en otros ámbitos. La profesión del trabajo social, te lo decía antes, tenemos un poco de complejo de inferioridad, sabemos mucho de nada y poco de todo. No tenemos una trayectoria de publicar, de formar nosotros. Y eso nos ha hecho perder terreno en un ámbito tan monopolizado por los psicólogos que lo hacen muy bien" (Segundo trabajador social entrevistado, comunicación personal, 27/1/2011, Cita de TS2B).

Las distintas variables se interconectan, como debe ser, y las conclusiones se vuelven complejas y más interesantes para el objetivo de este estudio:

"Con el grado a lo mejor. Eso se terminaría, pero como es una profesión que so mos aprendices de todo y maestros de nada, que yo la he elegido porque me gusta, yo no quiero ser maestra de nada" (Cita de TS6 M).

\section{0. - El LUGAR MÁS AUXILIAR QUE PERICIAL DEL TRA- BAJO SOCIAL JUDICIAL}

Esta variable conecta en cierta manera con la idea del trabajo social auxiliar más que pericial: ¿Dónde se sitúan los equipos psicosociales? si en una función más pericial o auxiliar de la justicia, el debate es amplio, aquí cabe introducirlo como parte de la influencia que puede tener en el supuesto previo analizado. ¿Cuáles son los conocimientos que precisamos para ser competentes en nuestro trabajo? ¿qué hay que saber para ser trabajador social de justicia? y si no somos peritos ¿dónde colocar la tarea de ayuda dentro de este ámbito tan reglamentado de la administración de justicia?

"Yo no soy un perito, yo soy un profesional de la administración de justicia, y como profesional de la administración de justicia desarrollo unas funciones y para desarrollar estas funciones necesito estar formada, y ya está. En cambio los psicólogos tienen la visión de que son peritos, y como son peritos judiciales tienen que tener un curriculum superextenso, cuando yo creo que no es necesario porque a mí se me pide que valore dinámicas familiares, entonces eso yo ya lo he aprendido y sí puedo aprender otras cosas relacionadas con ello, en cuanto que la sociedad evoluciona y hay que estar actualizado, pero ya está" (Tercer trabajador social de Barcelona entrevistado, comunicación personal, 28/1/2011, Cita de TS3B).

\section{1.- LA FALTA DE AUTOESTIMA PROFESIONAL}

Esta variable es ampliamente citada por psicólogos y trabajadores sociales, y al analizarla se aprecia en algunos testimonios, una valiosa autocrítica. Los profesionales del trabajo social se consideran capaces del trabajo en equipo y muy aptos para la práctica cercana al usuario. Cuando el trabajo requiere especialización se prefiere la derivación a otros profesionales y en el caso de sistematizar la práctica, investigar y teorizar sobre el trabajo diario, también se aprecia en los testimonios una peligrosa tendencia a la derivación.

"Nosotros aquí, la asistenta, la de las ayudas, la concepción social asimila ya por mucho que hayamos cambiado el nombre, la asistenta, en un sentido peyorativo, es la que te quita los niños, la que trae las ayudas y queda difuminado el otro trabajo que se está haciendo, a nivel profesional, y a altos ámbitos. Es una pena, pero yo creo que aquí también, nosotros también somos un poco res- 
ponsables de no habernos puesto las pilas y seguir" (Primer trabajador social de Barcelona entrevistado, comunicación personal, 27/ 1/2011, Cita de TS1B).

"Yo pienso que alguno de los trabajadores sociales que trabajamos habitualmente nos quedamos en el día a día y no estudiamos o damos docencia, en ese plano que hace público tu trabajo. Yo veo que hay muchos profesionales, y me incluyo, que se quedan en el trabajo diario, y eso se queda en el café, no sale fuera. Conozco más compañeros psicólogos que compaginan el trabajo diario en el juzgado con el de fuera" (Cita de TS1B).

La situación es peligrosa para la propia imagen de la profesión y sitúa la labor de los trabajadores sociales judiciales en un lugar peor del merecido. El trabajo realizado durante años en la administración de justicia debería haber generado un volumen importante de literatura. Pero no es sólo la cantidad sino también el lugar que esa investigación ocupa, en qué medida los otros profesionales citan y recurren a los textos de trabajo social judicial.

"Yo creo que es un error del trabajo social judicial y de otros. Nos gusta más el trabajo de campo, y lo que es el trabajo político, docente, investigador, un poco, lo tenemos más, no le damos importancia ni tiempo quizás porque no nos gusta. Pero indudablemente vas a una biblioteca y ves las publicaciones y lo ves, si vas buscando judicial o si buscas un tema que siempre ha sido nuestro, como trabajo social comunitario o servicios sociales. Los psicólogos llevan mucho tiempo haciendo un master en servicios sociales, y alucinas" (Tercer trabajador social de Barcelona entrevistado, comunicación personal, 28/1/ 2011, cita de TS3B).

"La profesión está encogida, nosotros nos infravaloramos, y esto es por los test o por tal o pascual. Yo creo que es verdad, nos tenemos que echar la culpa, de no hacernos valer, no para quedar por encima de nadie, pero cada uno desde su parcela y poner el trabajo social en el lugar que merece estar, tenemos que po- nernos las pilas para recoger lo que hacemos" (TS2B).

Los psicólogos se refieren a ello también, lo que refuerza el papel de esta variable, es interesante la mirada desde fuera para la creación de la propia imagen.

"Lo que pienso es que cuando se habla o se hacen estudios, yo no sé si en la parte de que los psicólogos opinan mucho, o que los trabajadores sociales no entran a opinar, no tengo muy claro, si sé que el psicólogo, algún psicólogo copa esa función. Yo no estoy de acuerdo ni lo comparto pero sí veo que en este ámbito concreto la función del trabajador social es un poco reducida" (Cita de PS3M).

"Yo creo que hay profesionales en general que tienen una ambición sana y mucha capacidad para decir quiero transmitir lo que sé, o lo que he trabajado hasta ahora, porque creo que es válida, cara a transferir conocimientos etc... y escribo libros y hago tesis. Todo lo que es un trabajo intelectual fuerte a nivel social y cultural. En el ámbito del psicólogo yo creo que sí se da, otra cosa es si se puede hacer" (GDPS).

"Yo he conocido muchas trabajadoras sociales y poca valentía en ellas para hacer un escrito, un debate, siempre un poco de reparo en meterse en estos temas. Ahora creo que hay más movimiento que hace años" (GDPS).

Algún trabajador social lo explica de forma metafórica y definitiva. Pero quizás la propia imagen no debe ser tan dependiente de las referencias externas, y dejar la adolescencia profesional en la que se encuentra el trabajo social, buscando la madurez en el conocimiento de las técnicas de investigación y en su puesta en práctica.

"Eso es como cuando tienes dos hijos, y a uno le dices lo guapo que es y al otro que es un torpe. Es lo del patito feo, te lo crees" (GDTS1). 


\section{2.- LA CONDICIÓN FEMENINA DEL TRABAJO SOCIAL}

Esta variable puede parecer polémica, pero se recogió en un debate de un grupo de discusión de trabajadores sociales, y por ello se apunta. El interés es que las profesionales que debatían eran mujeres y además de crear una variable tan discutible como interesante, ofrecían la solución y la esperanza en el futuro.

"¿Qué es lo que pasa también? Que hay muchas mujeres trabajadoras sociales" (GD TS1).

"Otra cosa, hay pocos trabajadores sociales hombres, pero luego son los más atrevidos" (GDTS1). TS1).

"Eso tiene que ver con el género" (GD

"El trabajo social es una profesión de mujeres. Tenemos que cambiar muchas cosas. Ya se han cambiado muchas cosas, no seamos pesimistas" (GDTS1).

\section{3.- LA MIRADA FUTURA HACIA UNA NUEVA FORMA- CIÓN, CON NUEVA TITULACIÓN (GRADO) Y NUEVOS CAMPOS}

Ya se han referido cuestiones relativas al futuro: a la nueva titulación, su nuevo plan de estudios y las posibilidades de una nueva sensibilidad hacia el trabajo social judicial en las instituciones del trabajo social.

"El tema de las prácticas, yo es un tema que le he dado vueltas siempre, me he sentido tentada de plantear. Igual que tienen prácticas los psicólogos, tenerlas. Yo estoy seguro, por ejemplo con la pública no, pero la vía de la Universidad Pontificia sería viable, aunque la pública a lo mejor también. Sería una for ma, desde el colegio verían, y si hay alumnos hablan de ti y conocen el trabajo. Hay master de Psicología Jurídica y no sé por qué no puede haber un "postgrado" de trabajo social jurídico" (Tercer trabajador social entrevistado, comunicación personal, 25/5/2011, Cita de TS3M).

\section{DIscusión}

Hay varias ideas claves para la discusión. En primer lugar es importante diferenciar la función pericial de la auxiliar en la función de los equipos psicosociales en España. La tarea pericial es meramente evaluadora e informativa para el juez, es aséptica, objetiva, ausente de intervención (psicológica o social). La función auxiliar comprende el asesoramiento al juez en todo el proceso (al margen de las partes y sus letrados), el seguimiento de la ejecución de la sentencia y la ayuda o intervención a la familia en cualquier aspecto educativo, asesor o de derivación a recursos. Esta distinción ha sido objeto de debate en España y tiene relación con las dos corrientes principales defensoras de discurso en este país. Los defensores de un discurso psicosocial no temen una función auxiliar amplia y con nuevos roles para los equipos psicosociales y los que creen en una actuación pericial pura defienden también un discurso psicológico forense.

Relacionado con esta primera idea, habría que discutir cómo este debate puede afectar a las familias usuarias de los servicios de los equipos psicosociales. Los profesionales que defienden un discurso psicosocial crean una nueva teoría mixta (hija del aporte psicológico y el aporte social) y centran su trabajo en la reinserción familiar. Los defensores de la Psicología Forense defienden un diagnóstico individual de cada miembro de la familia y creen y defienden que aportan más al juez en la medida en que distinguen cual es el progenitor más capacitado y cual el menos competente. Es evidente que la actuación profesional será diferente en ambos casos.

El tipo de discurso de los equipos psicosociales aporta alguna idea clave que se puede considerar y discutir: La idea de que los hijos deben estar con ambos padres, que los técnicos más antiguos de ambas profesiones en Barcelona apuntan. Es una idea importante como asegura Salzberg:

"es necesario garantizar la continuidad de las funciones materna y paterna y mantener el lugar de los hijos, estableciendo su contacto con el padre y con la madre" (1992, pp. 188-189). 
Y Garriga irá más allá:

"Para los hijos, sus padres siguen siempre juntos como padres. Se separan como pareja, a veces incluso mientras viven bajo el mismo techo, pero no se separan como padres" (2013, p. 169).

Otra idea es la distinta implicación de ambas profesiones para crear el discurso. La postura intelectual de Foucault de identificar el discurso con la fijación de una serie de normas y prescripciones para la creación de teorías o conceptos sobre una disciplina, puede valer para iniciar la búsqueda de documentación sobre profesionales que han creado teorías y conceptos y sobre instituciones profesionales que han configurado prescripciones y normas sobre cómo desarrollar estas teorías y conceptos. El resultado es abrumador: la mayoría de los textos que se pueden encontrar, para explicar la evaluación de familias en crisis matrimoniales, están escritos por psicólogos. La presencia de trabajadores sociales es menor (Alcazar, 2014; Alday et al., 2001; Couce, 2004: Hernández, 2002; Robles, 2004; Ruiz Rodríguez, 2004; Simón, 2010).

La profesora Brezmes incide en esta situación negativa del trabajo social como disciplina en relación a otras ciencias sociales.

"El Trabajo Social ha estado y está en una zona de penumbra en cuanto a su capacidad de influencia en otras Ciencias Sociales, produciéndose más que un diálogo casi un monólogo con ellas, puesto que las citas de Trabajo Social son escasísimas, si bien se inicia un camino importante" (2008, p. 107).

Pero cómo situarse dentro del equipo psicosocial en ese juego de distintas implicaciones y distintos poderes. Simón (2010) explica la situación de los equipos dentro de una gran variedad en la que nadie ha expuesto un protocolo central de trabajo en el que inspirarse y por ello está en construcción.

"Derivado de la dispar conformación de los equipos de trabajo definidos para dar respuesta a periciales, en la práctica se llevan a cabo diferentes procedimientos de trabajo que van tanto desde una respuesta interdisci- plinar como multidisciplinar hasta la individual" (p. 183).

Y da su conclusión acerca de cómo debe entenderse el trabajo:

"La labor pericial de cada disciplina supone un enriquecimiento $\mathrm{y}$, al mismo tiempo, es una constante fuente de discusión-reflexión que sería deseable se produjera en la valoración de todos los dictámenes periciales y no como algo puntual" (p. 183).

Otra idea importante es la derivada de la importancia de la formación previa, las distintas titulaciones, lo que va a cambiar con el cambio a la titulación de grado para ambas profesiones.

Ponce de León refiere el avance que va a suponer para la profesión del trabajador social el cambio al grado en la titulación académica, no sólo respecto a la comparación con otras profesiones o disciplinas, también en lo que atañe a la carrera académica y a la investigación sobre la práctica.

"Después de año de esfuerzos, finalmente se ha aprobado la titulación de grado en Trabajo Social que nos permite equipararnos al resto de titulaciones posteriores y nos facilita una mejor y más amplia formación. Pero también nos permite realizar investigaciones sobre nuestra materia de trabajo, que hasta ahora estaban en manos de otros profesionales de las Ciencias Sociales. Todo el proceso generará un mayor conocimiento de las necesidades y expectativas de la población en general, que nos hace intuir una nueva etapa de madurez intelectual y profesional en el trabajo social" (2011, p. 119).

"El título de grado en Trabajo Social nos ha permitido la equiparación de los estudios con el resto de Europa, así como el reconocimiento profesional, con la posibilidad de continuar enriqueciéndonos con los estudios de postgrado y a su vez acceder a una variada oferta educativa y laboral en otros países alejados del nuestro, además de enfrentarnos al desafío que suponen las nuevas tecnologías como una gran aliada para avanzar con mayor rapidez" (2011, p. 123). 
Otra discusión importante puede ser la de considerar que hay una situación real de disputa entre la multidisciplinariedad, como yuxtaposición de disciplinas, y la interdisciplinariedad como mezcla de ella en un nuevo axioma (Ander-Egg \& Follari, 1988), la cual está perdiendo la batalla por la escasez de recursos y el uso indiscriminado de profesionales, sin considerar su origen académico.

Como conclusión a esta discusión, hay que decir que este artículo informa una investigación general en la que se ha realizado un estudio cualitativo de los discursos de profesionales. La elección de las ciudades de mayor población y mayor densidad demográfica ha sido acertada, pero ha quedado pendiente recuperar el discurso del resto de provincias españolas. Ese es un estudio por hacer, necesario pero más costoso a todos los niveles, lo que haría necesario un aporte externo de recursos, más allá de los recursos personales de un investigador doctoral en una investigación básica como la presente. La elección de métodos cualitativos es más adecuada en la medida que los discursos han partido de profesionales, lo que ha generado una documentación rica que ha permitido generalizar y saturar el campo, generando teoría sobre ese discurso o discursos profesionales.

\section{Conclusiones}

La lectura detallada de los discursos y su agrupación en distintas variables permite confirmar, asegurar el supuesto previo y avanzar conclusiones sobre las distintas variables que aparecen interconectadas y referidas a esa mayor presencia de la Psicología Forense y a la casi ausencia de discurso de la profesión del trabajo social judicial.

Los profesionales de Barcelona plantean una historia común de lo psicosocial y una creencia inicial en que la práctica de la profesión debía partir de ambas profesiones. La orientación psicosocial se une en el discurrir de la historia profesional con otra corriente psicológica diferente y con una explicación distinta de la tarea judicial psicosocial.
Desde las dos profesiones se incide en la escasez de creadores de discurso y en el planteo aislado de cada propuesta, sin puesta en común ni debate general.

Estas variables iniciales se citan en los relatos profesionales desde una necesidad de explicar el estado de la situación. A partir de ahí se aprecia una intención exploratoria y se desarrollan las distintas cuestiones que rodean esta realidad profesional. La imagen pública de las profesiones sería el primer aspecto que se desarrolla y no sólo se describe la supuesta menor valoración de la profesión del trabajador social, sino que también se cuestiona su utilidad si no es capaz de evolucionar en el contenido de su aportación pericial.

Las instituciones del trabajo social es otro de los temas comunes que surge en los relatos: la distinta forma de considerar lo judicial desde los colegios profesionales o las facultades de psicología a las mismas instituciones en el trabajo social. La comparación destaca la escasa atención de estas últimas a la práctica psicosocial en el ámbito de justicia.

Unido a lo anterior, se encuentra la variable formativa, la ausencia de discurso teórico del trabajo social judicial proviene de una escasa sistematización de la práctica, pero también de una ausencia destacada de los planes de estudio de esta disciplina.

La profesión del trabajo social es generalista, se ocupa de gran cantidad de sectores y su formación es ancha pero no larga. La función pericial se apoya en la especialización, lo que crea problemas si el profesional no adquiere esa capacidad que la realidad académica no le ha provisto. También se destaca la menor categoría académica del trabajo social, cuestión que ya se ha igualado con el establecimiento del grado y que debe actualizarse en lo profesional, más allá de lo académico. Todo ello puede llevar a una creencia menor del rol profesional, a una deficiente autoestima profesional. Alguna trabajadora social introduce la variable género en su interpretación, aquí sólo se apunta ya que daría lugar a un debate profundo por sí misma.

Otra cuestión destacable es la de la creencia extendida entre los trabajadores sociales judiciales de ser una figura auxiliar del juez, 
no tanto pericial como de ayuda. Esto incrementa el debate e introduce una nueva variable en juego que complica la interpretación del rol.

La mirada hacia el futuro surge con dudas y con nuevas tareas y campos, también con una nueva titulación.

\section{REFERENCIAS BIBLIOGRÁFICAS}

Alcázar, R. (2014). Diseño de una escala para la evaluación de la custodia compartida en el ámbito judicial (ICC) [A scale design for the assessment of joint custody in family court (ICC)]. Azarbe, 3, 271-277.

Alday, M.A., Ramljak, N.L. \& Nicolini, G. (2001). El trabajo social en el servicio de justicia [Social work at the department of justice]. Buenos Aires: Espacio.

Ander-Egg, E. \& Follari, R. (1988). Trabajo social e interdisciplinariedad [Social work and interdisciplinary]. Buenos Aires: Humanitas.

Bolaños, J.I. (1993). El proces contencios de separación i/o divorci: Una visión psicosocial [The process of separation contencios and divorce: A psychosocial view]. Studia Jurídica, $5,129-142$.

Brezmes, M. (2008). El trabajo social en España [Social work in Spain]. Murcia: Editum.

Camacho, I., Arroyo, R. \& Serrano, M. (2011). Psicología y lógica: Una relación transdisciplinaria [Psychology and logic: A transdisciplinary relation]. Interdisciplinaria, 28(2), 221-230.

Catalán, M.J. (1999). El informe psicológico en separaciones familiares: Cuestiones éticas [The psychological report in family separations: Ethical issues]. Papeles del Psicólogo, Junio, 73.

Chambon, A.S., Irving, A. \& Epstein, L. (2001). Foucault y el trabajo social [Foucault and social work]. Granada: Maristán.

Couce, P. (2004). El dictamen pericial social de los trabajadores sociales [The social expert opinion of the Social Workers]. Trabajo pre - sentado en el Congreso Nacional de Profesionales de la Pericia Judicial. Valencia. España.

De Andrés, A. (1997). Intervención sociojurídica con familias en conflicto. En García, L. (Ed.), El derecho y los servicios sociales [The law and social services] (pp. 114-143). Granada: Comares.

Fariña, F., Arce, R. \& Sotelo, A. (2010). ¿Es efectivo el estudio psicométrico estándar del peritaje del estado clínico y de la disimulación en progenitores en litigio por la guarda y custodia de menores? [How effective is the standard psychometric study of expertise of clinical status and dissimulation in litigation parents in the care and custody?]. Revista Iberoamericana de Psicología y Salud, 1(1), 65-79.

Garriga, J. (2013). El buen amor en la pareja [The great love in marriage]. Barcelona: Destino.

Gómez, F. (1988). El trabajo social en la adopción [Social work in the adoption]. Cuadernos de Trabajo Social, 1, 213-218.

Gómez, F. \& Soto, R. (2015a). El trabajador social de la administración de justicia española en los procesos de rupturas matrimoniales [Social worker performance in the Spanish administration of justice on marital breakdown processes]. Estudios Socio-Jurídicos, 17(2), 197-232. http://dx.doi.org/10.12804/esj17.02. 2015.06

Gómez, F. \& Soto, R. (2015b). Nuevas tareas, nuevos lugares del trabajo social judicial en España [New tasks, new places of justice social work in Spain]. Revista Serviço Social \& Sociedade, 121, 10-23. http://dx.doi.org/10.15 90/0101-6628.016

Hernández, A. (2002). Dictamen pericial de los trabajadores sociales [Expert opinion of the social workers]. Revista La Toga, 133 (enerofebrero), 16-18. Colegio de Abogados de Sevilla.

Ibáñez, V. \& De Luis, P. (Junio, 1987). Juzgados de familia: Psicología jurídica o justicia psicológica [Family courts: Legal psychology or psychological justice]. Papeles del Psicólogo, 30, 10-13. 
Ponce de León, L. (2011). Investigación y trabajo social: Investigando en el pasado las claves del futuro de la profesión [Research and social work: Researching last key to the future of the profession]. Trabajo presentado en el $5^{\circ}$ Congreso de Trabajo Social de Madrid. Colegio Oficial de Trabajadores Sociales de Madrid.

Ponce de León, A. \& Krmpotic, C. (2012). Traba jo social forense. Balance y perspectivas [Forensic social work. Balance and perspectives]. Buenos Aires: Espacio Editorial.

Quintero, A.M. (2010). Pruebas psicosociales en derecho de infancia, adolescencia y familia [Psychosocial tests right to childhood, adolescence and family]. Informe final de investigación. Colombia: Universidad de Antioquía.

Real Academia Española. (2015). Diccionario de la lengua española [Spanish dictionary], (23 ed.). Madrid, España. Recuperado el 11 de mayo de 2015 de http://www.rae.es/

Ruiz Rodríguez, P. (2004). El trabajador social como perito judicial [The social worker like court expert]. Zaragoza: Certeza.
Robles, C. (2004). La intervención pericial en trabajo social [The expert intervention in social work]. Buenos Aires: Espacio.

Salzberg, B. (1992). Los niños no se divorcian [Children do not get divorced]. Barcelona: Logos.

Sierra Murguía, M.A. (2013). El rol del psicólogo en el equipo interdisciplinario de cirugía bariátrica [The psychologist's role in a bariatric interdisciplinary team]. Interdisciplinaria, 30 (2), 191-199.

Simón, M. (2010). Aportaciones del trabajo social a la pericial de familia. En J.J. Tapia (Dir.), Custodia compartida y protección de menores [Custody and protection of minors] (pp. 180192). Madrid: Dykinson.

Valles, M. (2007). La grounded theory y el análisis cualitativo por ordenador. En M. García, J. Ibá ñez \& F. Alvira (Comps.), El análisis de la realidad social. Métodos y técnicas de investigación [The analysis of social reality. Methods and research techniques], (3ra. ed., pp. 575-603). Madrid: Alianza.

Facultad de Trabajo Social Universidad Complutense de Madrid Madrid - España

Fecha de recepción: 21 de agosto de 2015 Fecha de aceptación: 4 de enero de 2016 